THE ASTROPHYSICAL JOURNAL SUPPLEMENT SERIES, 90:841-844, 1994 February

(C) 1994. The American Astronomical Society. All rights reserved. Printed in U.S.A.

\title{
EVOLUTION OF SUPERNOVA REMNANTS WITH COSMIC RAYS AND RADIATIVE COOLING
}

\author{
E. A. DORFI \\ Institut für Astronomie, Universität Wien, Türkenschanzstrasse 17, A-1180 Wien, Austria \\ Received 1993 March 8; accepted 1993 July 14
}

\begin{abstract}
Recent numerical models for SNR evolution are presented, including first-order Fermi acceleration with injection of suprathermal particles at the shock wave, heating due to dissipation of Alfvén waves in the precursor region and radiative cooling of the thermal plasma. The X-ray fluxes obtained from these SNR models show significant differences depending on the acceleration efficiency of cosmic rays. $\gamma$-ray fluxes are calculated originating from $\pi^{0}$-decay of pions generated by collisions of the high-energy particles with the thermal plasma. Cooling of the thermal plasma and dissipation of Alfvén waves in the precursor are important to determine the final amount of the explosion energy $E_{\mathrm{SN}}$ which is transferred into cosmic rays.
\end{abstract}

Subject headings: acceleration of particles — cosmic rays - gamma rays: theory - shock waves supernova remnants

\section{INTRODUCTION}

It is commonly accepted that the galactic component of the cosmic rays is accelerated in shock waves originating from supernova explosions (e.g., Blandford 1988; Drury et al. 1989). These charged particles gain energy in a first-order Fermi process (Axford et al. 1977; Krymsky 1977; Bell 1978a, b; Blandford \& Ostriker 1978) which can be treated by an additional energy equation for the cosmic rays if averaged over the particle distribution function (e.g., Drury 1983). In this case the effective diffusion coefficient $\bar{\kappa}$ as well as $\gamma_{c}$ the adiabatic index of cosmic rays have to be estimated dynamically during the SNR evolution according to an approximative treatment following Drury et al. (1989). Since these high-energy particles provide a major contribution to the energy balance of the interstellar medium they have to be included in the calculation of interstellar dynamical phenomena. The presented models of SNRs include the process of particle acceleration in shock waves, the injection of suprathermal particles, heating by dissipation of Alfvén waves in regions of large cosmic-ray gradients and radiative cooling of the thermal plasma. The resulting system of nonlinear PDEs is solved fully implicit on an adaptive grid to ensure the necessary numerical accuracy during the computations (Dorfi 1990, 1991). The shock structure can be modified in a nonlinear way by the acceleration energetic particles leading to smaller postshock gas temperatures and a different thermal history of the thermal plasma. Therefore we expect also that the X-ray emission from the SNR depends on the efficiency of the acceleration process. Since the acceleration of high-energy particles is closely connected to the production of $\gamma$-ray photons above $100 \mathrm{MeV}$ (e.g., Stecker 1973, Stephens \& Badhwar 1981) collisions of the cosmic-ray protons with the thermal plasma create neutral pions and these pions decay into observable $\gamma$-quanta. Hence, the observed $\gamma$-ray flux above $100 \mathrm{MeV}$ depends on the cosmic-ray energy density times the gas density.

\section{EFFECTS OF RADIATIVE COOLING AND PARTICLE ACCELERATION ON THE SNR EVOLUTION}

The usual system of time-dependent cosmic rays hydrodynamics is applied in spherical symmetry and the cosmic rays are also treated on this level averaged over the particle distribution function (Dorfi 1990; Jones \& Kang 1990). It is assumed that the SN explodes into a homogeneous medium and modifications of the external medium due to a possible stellar wind from a SNprogenitor are not taken into account. The supernova explosion energy is set to $E_{\mathrm{SN}}=10^{51} \mathrm{ergs}$, the ejected mass to $M_{e}=5 M_{\odot}$. The external density of $n_{0}=0.3 \mathrm{~cm}^{-3}$ and the temperature of $T_{\text {ext }}=8000 \mathrm{~K}$ correspond to the warm interstellar medium.

The time evolution of the different energy contributions is shown on a linear scale up to $7 \times 10^{5} \mathrm{yr}$ in Figure 1 . The case depicted in Figure $1 a$ is characterized by a low conversion efficiency from the explosion energy $E_{\mathrm{SN}}$ to the total cosmic-ray energy $E_{\mathrm{cr}}$ and $E_{\mathrm{cr}}$ stays below $0.04 E_{\mathrm{SN}}$ during the entire evolution. Cooling effects become important after $4.4 \times 10^{4} \mathrm{yr}$ (e.g., Kahn 1976). We emphasize the importance of radiative cooling where the most of the SNR thermal energy content is already radiated away after $\sim 5 \times 10^{4} \mathrm{yr}$, and $E_{\text {cool }}$ denotes the remaining SNR energy. Figure $1 b$ exhibits the temporal dependence of the energies in the case of a more efficient cosmic-ray production. This model includes also heating of Alfvén waves in the precursor region of the forward shock (written like $\alpha_{\mathrm{H}} v_{\mathrm{A}}\left|\nabla P_{c}\right|, \alpha_{\mathrm{H}}=1$; e.g., Völk et al. 1984) which yields an increase of the thermal energy $E_{t h}$, although radiative cooling decreases the total energy of the remnant.

The injection of particles at the shock is given by $\epsilon_{\text {inj }}=10^{-3}$, i.e., the fraction $\epsilon_{\text {inj }}$ of the incoming kinetic energy is carried off by the cosmic rays (Drury 1983). For higher values of $E_{\mathrm{cr}}$ (cf. Fig. $1 b$ ) cooling becomes important at earlier times because the thermal gas remains cooler during the Sedov-phase. We can estimate the onset of cooling at $3.4 \times 10^{4} \mathrm{yr}$ but $E_{\text {cool }}$ decreases more slowly in time compared to Fig. $1 a$. 

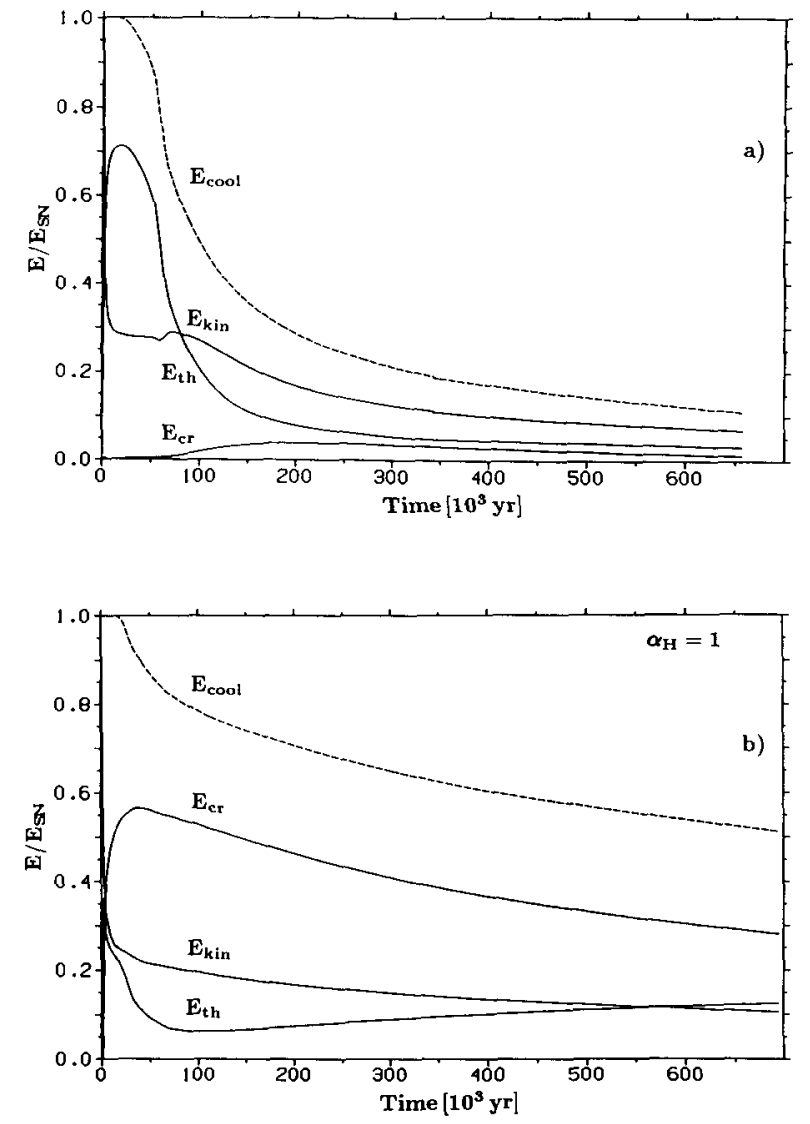

FiG. 1.-The temporal evolution of the total energies as a function of time in units of the initial SN explosion energy $E_{\mathrm{SN}}$ in the case $(a)$ of low cosmic-ray production and $(b)$ for a higher conversion of $E_{\mathrm{SN}}$ into $E_{\mathrm{cr}}$. Case $(b)$ includes Alfvénic heating and injection of particles.

Radiative cooling alters also the radial structure in the cooling region behind the forward shock and can lead to the development of so-called secondary shocks (Falle 1975, 1981). In the case of energetic particles we have an additional pressure which limits catastrophic cooling and allows a smooth expansion of the forward shock. In Figure 2 we present the density (panel $a$ ), the velocity (panel $b$ ), the cosmic-ray energy (panel $c$ ), and the gas temperature (panel $d$ ) in the shock frame for the model depicted also in Figure $1 b$. Note that the cosmic-ray pressure inside the remnant is about two orders of magnitude above the external value. The density in units of the external density of $n_{0}=0.3 \mathrm{~cm}^{-3}$ reveals the increase by cooling but also a small cosmic-ray precursor infront of the shock. The temperature (Fig. $2 d$ ) exhibits the almost isothermal shock wave, and we can trace the cooling front moving inward relative to the shock frame. We want to emphasize the density enhancement due to cooling and the occurrence of complicated structures in the downstream region of the shock wave which yield also fluctuations in the collisional production of neutral pions and the associated $\gamma$-ray fluxes. In all computations carried out the compression ratio $r$ of the radiative shock wave remains below $r \leqslant 70$ limited by the downstream cosmic ray pressure gradients. The postshock velocity is still of the order of $100 \mathrm{~km} \mathrm{~s}^{-1}$ showing a cosmic-ray precursor growing in time (cf. Fig. 2b).

\section{X-RAY AND $\gamma$-RAY FLUXES}

During their whole evolution SNRs contain hot gas which radiates at X-ray temperatures. Since the plasma is heated by several shock waves other nonthermal processes occurring at the shock transition can alter the amount of explosion energy transferred into the radiating gas. In particular, the process of particle acceleration in shock waves can be very efficient in certain parameter regimes and convert almost all incoming kinetic energy to cosmic rays (Axford 1981; Drury \& Völk 1981). A more detailed discussion of these effects can be found in Dorfi \& Böhringer ( 1993).

Most of the $\gamma$-rays above $100 \mathrm{MeV}$ are due to the decay of neutral pions created by collisions of cosmic-ray proton in the energy range between 1 and $30 \mathrm{GeV}$ with the thermal plasma. Taking the produced cosmic-ray spectrum similar to the observed one a yielding factor of $q_{\gamma}=1.4 \times 10^{-13} \mathrm{~cm}^{3} \mathrm{ergs}^{-1} \mathrm{~s}^{-1}$ for the production of $\gamma$-rays above $100 \mathrm{MeV}$ by $\pi^{0}$-decay can be calculated (Higdon \& Lingenfelter 1975). The $\gamma$-ray flux is then given by

$$
F_{\gamma, \mathrm{SNR}}=\frac{q_{\gamma}}{4 \pi d^{2}} \int_{0}^{R_{s}} n E_{c} 4 \pi r^{2} d r
$$

where $R_{s}$ denotes the radius of the shock front, $d$ the distance to the SNR, $n$ the gas number density and $E_{c}$ the cosmic-ray energy density.

Secondary shocks triggered by radiative cooling decrease the cosmic-ray energy density as well as the gas density in the vicinity of the forward shock, yielding a lower $\gamma$-ray flux $F_{\gamma, \mathrm{SNR}}$. The case of higher cosmic-ray pressures produces an enhancement of the $\gamma$-ray fluxes already at earlier times (compared to the low cosmic-ray case) but small-scale shock waves in the downstream region cause more visible fluctuations on the $\pi^{0}$-production because the cooler gas is pushed more easily by the cosmic-ray pressure gradients. In this case we get also an important contribution of creating neutral pions in the precursor region of the forward shock. Since Alfvénic heating in the precursor region is included, we observe a faster decrease in time of the $\gamma$-ray flux. From the smooth expansion of the shock radius we can conclude that radiative cooling does not affect the motion of the shock front compared to simulations without cosmic rays (Falle 1975, 1981).

Figure 3 depicts the evolution of the X-ray flux between 0.1 $\mathrm{keV}$ and $2.4 \mathrm{keV}$ as a function of time. The two curves in this plot correspond to the two remnants differing by the production of energetic particles. After $t \gtrsim t_{\mathrm{sw}}$ (here: $t \gtrsim 10^{11} \mathrm{~s}$ ) the remnant of case b) (effective cosmic-ray acceleration) starts already cooling which increases the $\mathrm{X}$-ray flux in this energy range. The maximum is reached at $\sim 1.6 \times 10^{4} \mathrm{yr}$, whereas the case with no significant cosmic rays (case $a$, upper curves at later times) exhibits its maximum around $3.8 \times 10^{4}$ years which is a factor of 4 more luminous than case b). After that event, both $X$-ray fluxes decrease almost parallel as seen in Fig. 3 , i.e., $t \gtrsim 10^{12} \mathrm{~s}$ and the relative difference is given by about a factor of 30 . Hence, from this figure it follows that the efficiency of particle acceleration can be traced indirectly in the $\mathrm{X}$-rays originating from the thermal plasma. 

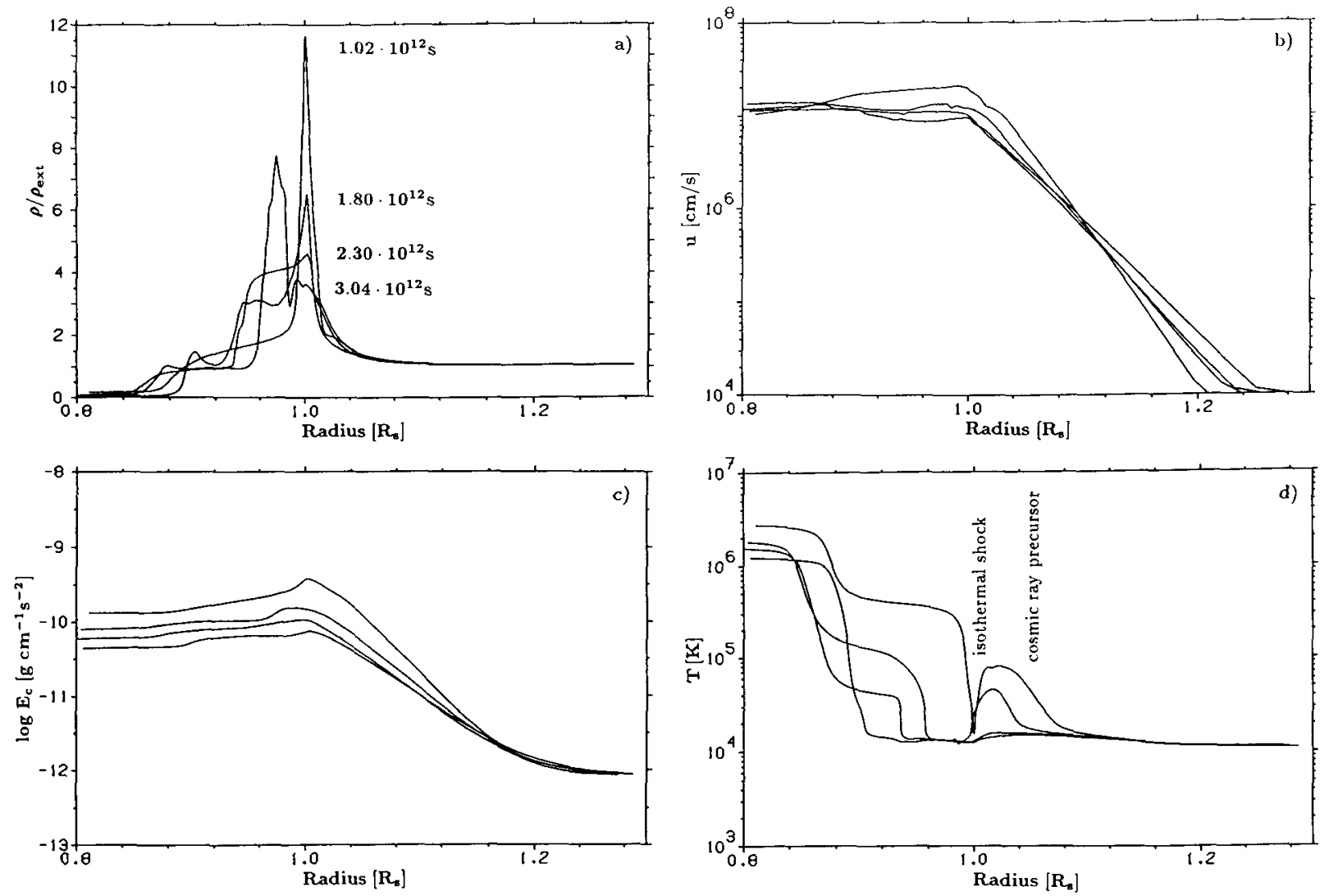

FIG. 2.-The radial structure of the physical variables in the shock frame in units of the shock radius $R_{\mathrm{s}}$ between $10^{12} \mathrm{~s}$ and $310^{12} \mathrm{~s}$, showing the development of downstream features due to radiative cooling.

\section{DISCUSSION AND CONCLUSIONS}

From the calculations presented it is clear that radiative cooling is important to determine the amount of supernova energy $E_{\mathrm{SN}}$ converted into cosmic rays. The models of SNR evolution including cooling can be responsible for the production of the galactic cosmic rays up to particle energies of about $10^{14} \mathrm{eV}$

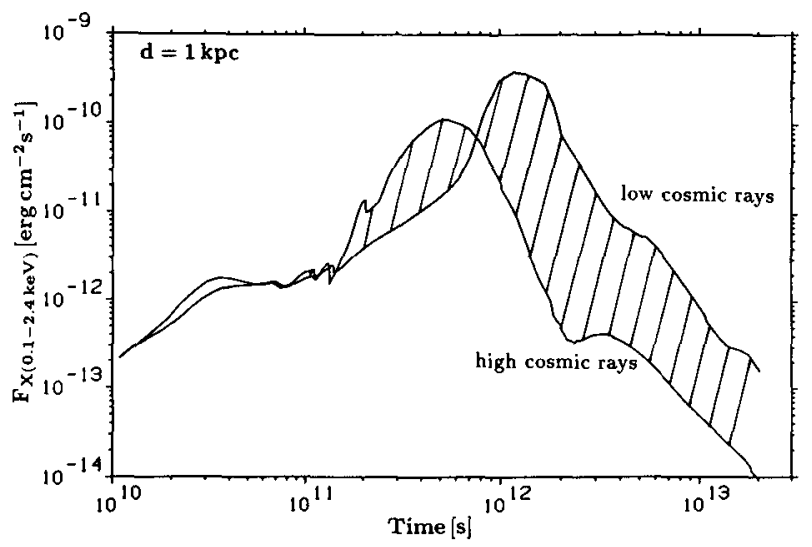

Fig. 3.-The flux in the $0.1-2.4 \mathrm{keV}$-range as a function of time. The upper curve (at later times) shows the case of low cosmic rays yielding larger X-ray fluxes by about a factor of 30 compared to the remnant with large amounts of cosmic rays. nucleon $^{-1}$. The dissipation of Alfvén waves in the precursor of the forward shock plays an important role, in particular during the late phases of the SNR evolution because the thermal energy $E_{\mathrm{th}}$ can remain almost constant in time during the cooling phase. The motion of the shock front is influenced by the highenergy particles since the downstream total pressure gradient cannot drop below the cosmic-ray pressure gradient. Moreover, the compression ratios are limited by cosmic rays and we find no "catastrophic" cooling associated with a dramatic enhancements of the density. In all SNR simulations reported the compression ratio is limited to $r \leqslant 70$. The X-ray flux from an SNR can differ by up to a factor of 30 depending on the efficiency of particle acceleration. The presence of cosmic rays leads to the production of $\gamma$-ray photons with energies above $100 \mathrm{MeV}$, and we end up at most with $F_{\gamma, \mathrm{SNR}} \simeq 10^{-6}$ photons $\mathrm{cm}^{-2} \mathrm{~s}^{-1}$ scaled to a distance of $d=1 \mathrm{kpc}$. Secondary shocks change the $\gamma$-ray fluxes by modifying the cosmic-ray energy density in the downstream region of the forward shock. These fluctuations in the $\gamma$-ray flux are expected to be of the order of the sound crossing time in the cooling region.

Since these SNR models are calculated in spherical symmetry no quantitative estimates can be given on local (nonradial) inhomogeneities. Nevertheless, a few points modifying the conclusions should be addressed. First, a SN explosion into a cloudy medium (e.g., KcKee \& Ostriker 1977) can be different in the case of cosmic-ray production because less thermal energy is available to evaporate clouds by reducing the conduc- 
tion from the hot phase to the cold clouds. Second, such embedded clouds which can survive more easily in SNR accelerating cosmic rays will also increase the local $\gamma$-ray emission due to the higher densities of the clouds. Furthermore, the $\gamma$-luminosity of these clouds can be enhanced by bremsstrahlung from secondary electrons (Morfill 1982a, b). Both effects can act together with an increased mean density due to the mass load from interstellar clouds and hence the $\gamma$-fluxes stated above can be seen as lower limits.

It is clear that the X-ray appearance of a SNR is altered in the case of cosmic-ray production because less thermal gas is able to radiate in the $\mathrm{X}$-ray range. A more detailed description can be found in Dorfi \& Böhringer (1993) and will therefore be omitted here. The structure of a shock accelerating particles is modified by a precursor generated by the upstream diffusion of energetic particles. In this precursor region the incoming plasma is already decelerated before entering the so-called subshock, and we get a heating of the thermal gas infront of the shock front. This mechanism has been used by Morfill, Aschenbach, \& Drury (1984) to explain the fainter halo around the Cas A SNR which extends beyond the suggested gas shock. However, in the models presented the plasma is heated up in the precursor region only to temperatures without a significant $\mathrm{X}$-ray emission. A different temporal evolution of the mean cosmic-ray diffusion coefficient $\bar{\kappa}$ which has been calculated according to Drury et al. (1989) can lead to a more pronounced X-ray structure ahead of the gas shock. Summarizing the results of a number of numerical calculation on SNRs with particle acceleration and radiative cooling only high-resolution observations on an SNR may be able to disentangle the modifications of the shock front caused by particle acceleration and by expansion into a nonhomogeneous medium. Taking the calculated X-ray fluxes it is impossible to decide which case of low or high cosmic-ray production is preferred, and we have to await more detailed calculations, including also the time-dependent ionization in a SNR to obtain X-ray spectra which could demonstrate a clearer evidence in certain spectral windows for particle acceleration in SNRs.

I would like to thank the referee G. E. Morfill for his clarifying comments on the manuscript. Part of this work is supported by the Jubiläumsfond der Österreichischen Nationalbank under project number 4605 .

\section{REFERENCES}

Axford, W. I. 1981, in Proc. Internat. School and Workshop on Plasma Astrophysics (Varenna), ESA SP-161, 425

Axford, W. I., Leer, E., Skadron, G. 1977, Proc. 15th Internat. CosmicRay Conf. (Plodiv), 11, 132

Bell, A. R. 1978a, MNRAS, 182, 147 1978b, MNRAS, 182,443

Blandford, R. D. 1988, in Supernova Remnants and the Interstellar Medium, ed. R. S. Roger \& T. L. Landecker (Cambridge Univ. Press), 309 Blandford, R. D., \& Ostriker, J. P. 1978, ApJ, 221, L29

Dorfi, E. A. 1990, A\&A, 234, 419

- 1991, A\&A, 251, 597

Dorfi, E. A., \& Böhringer, H. 1993, A\&A, 273, 251

Drury, L. O'C. 1983, Rep. Prog. Phys., 46, 973

Drury, L. O'C., Markiewicz, W., \& Völk, H. J. 1989, A\&A, 225, 179

Drury, L. O'C., \& Völk, H. J. 1981, ApJ, 248, 344
Falle, S. A. E. G. 1975, MNRAS, 172, 55 1981, MNRAS, 195, 1011

Higdon, J. C., \& Lingenfelter, R. E. 1975, ApJ, 198, L17

Jones, T. W., \& Kang, H. 1990, ApJ, 363, 488

Kahn, F. D. 1976, A\&A, 50, 145

Krymsky, G. F. 1977, Dokl. Nauk. SSR, 234, 1306 (Engl. trans., Soviet Phys. Dokl. 23, 327)

McKee, C. F., \& Ostriker, J. P. 1977, ApJ218, 148

Morfill, G. E. 1982a, MNRAS, 198, 583 $1982 \mathrm{~b}, \mathrm{ApJ}, 262,749$

Morfill, G. E., Aschenbach, B., \& Drury, L. O'C. 1984, Nature, 311, 358 Stecker, F. W. 1973, ApJ, 185, 499

Stephens, S. A., \& Badhwar, G. D. 1981, Ap\&SS, 76, 213

Völk, H. J., Drury, L. O'C., \& McKenzie, J. F. 1984, A\&A, 130, 19 